\title{
EL EMPODERAMIENTO DE LA MUJER EN JORDANIA A TRAVÉS DE MICROCRÉDITOS PARA SU EMPRENDIMIENTO LABORAL ${ }^{1}$
}

\author{
María del Carmen Hidalgo-Chacón Díez ${ }^{2}$
}

El empoderamiento de la mujer en Jordania a través de microcréditos para su emprendimiento laboral.

Resumen: El objetivo del presente artículo es mostrar una propuesta de cooperación entre una Organización Internacional y el Ministerio de Asuntos Sociales de Jordania para fomentar el empoderamiento de la mujer jordana a través de microempresas familiares tales como confección, artesanía, educación, cuidado de niños de otras mujeres trabajadoras y cooperativas de pan y dulces tradicionales. Para conseguir este objetivo, el artículo se ha dividido en dos partes: una primera donde se analiza brevemente la situación laboral de la mujer árabe, y más concretamente de la mujer en Jordania, y una segunda parte que es la descripción de la propuesta de cooperación.

Palabras claves: Empoderamiento de mujer, Jordania, zona MENA, microempresas familiares, emprendimiento laboral, cooperación internacional.

\section{Women Empowerment in Jordan through Microcredits for their Entrepreneurship.}

Abstract: The purpose of the following article is a proposal of cooperation between an International Organization and the Ministry of Social Development of Jordan for boosting the empowerment of Jordanian women through familiar microenterprises (dressmaking, crafts, education: after school learning centre, kids caring, and cooperative for bread and traditional sweets). The article contains two parts: the first one analyses briefly the situation of Arabic women, particularly in Jordan, as a labour force, and the second part is the proposal of cooperation per se.

Key words: Women's empowerment, Jordan, MENA region, familiar microenterprises, entrepreneurship, cooperation international

\section{Introducción}

Actualmente nos encontramos ante un mundo globalizado que está favoreciendo

la eliminación de la confrontación entre pueblos y sociedades. En este marco se incluye

\footnotetext{
${ }^{1}$ Fecha de recepción: 17/10/2019.

Fecha de aceptación: 30/12/2019.

2 Doctora en Estudios Semíticos por el Centro de Estudios de Asia Central y Oriente Medio (CNMS), Universidad de Marburg, Alemania y Máster en Relaciones Internacionales por la Universidad Rey Juan Carlos, España; $\square$ mcarmen.hidalgo-chacon@hotmail.com.
} 
también la lucha por la igualdad de género y el empoderamiento social y económico de la mujer ya que esta forma parte esencial de la sociedad y está tan cualificada como el hombre para realizar las mismas labores.

Sin embargo, en los países árabes, la mujer sigue sufriendo una clara discriminación con respecto al hombre en cuanto al tema laboral y social se refiere. A pesar de que todos los países árabes firmaron y ratificaron en su día la Convención para la Eliminación de Toda Forma de Discriminación contra La Mujer, conocido como CEDAW por sus siglas en inglés (ONU 1981: 13-143), con reservas de ciertos $\operatorname{artículos}^{3}$, la discriminación sigue existiendo. Un ejemplo de ello son las barreras laborales (como veremos en el epígrafe 2.1.) que impiden a las mujeres competir en igualdad de condiciones con los hombres y más difícil aún acceder a un puesto de alta responsabilidad política o financiera.

Jordania, país en el que centramos nuestra propuesta, no es una excepción, puesto que adolece de los males sociales y legales (como veremos en el epígrafe 2.2.) que afectan a la mayoría de las mujeres árabes, si bien es cierto que cuentan con más derechos que otros países fronterizos como es Arabia Saudí, donde hasta no hace mucho a la mujer no se les estaba permitido conducir.

Por tanto, el presente trabajo tiene como finalidad ofrecer propuestas de microempresas familiares que estarán dirigidas por mujeres emprendedoras y financiadas a través de microcréditos para trabajos de confección, artesanía, pequeñas academias, cuidado de niños y cooperativas de pan y dulce. Se plantea como el primer

\footnotetext{
${ }^{3}$ La mayoría de los países se reservaron los artículos siguientes: Artículo 2, Artículo 9 (2), Artículo 15 y Artículo 16. Túnez y Yibuti no hicieron ninguna reserva (ver OCDE/CAWTAR 2014a: 44).
} 
paso de un futuro proceso de cooperación internacional entre una Organización Internacional y el Ministerio de Desarrollo Social de Jordania.

En definitiva, con estas propuestas se pretende fomentar el espíritu empresarial de las mujeres jordanas, empoderarlas económicamente, promover su acceso a un trabajo digno, aumentar sus capacidades, sus oportunidades y su inclusión en el mercado laboral para que contribuyan a la economía familiar y, además, motivarlas a adquirir independencia e incrementar su bienestar social y económico como principio internacional de derechos humanos.

\section{Visión general de la mujer árabe como fuerza laboral. El caso de la mujer en} Jordania

Durante todo el siglo pasado la condición de la mujer mejoró considerablemente con el surgimiento de asociaciones de mujeres que jugaron un papel fundamental en el desarrollo de los derechos humanos y el antiautoritarismo, entre las que podemos destacar la Asociación Intelectual de Mujeres fundada en Egipto en 1914 por Huda Shaarawi, aristócrata que trabajó para la educación y derechos de las mujeres y que logró convocar a un gran número de mujeres en 1919 en las movilizaciones que reclamaban la independencia de Gran Bretaña, lo que supuso una defensa en su condición de ciudadanas reclamando la libertad, "una libertad colectiva, pero también personal" (Paradela 2015: 20-21). Asimismo, en Palestina se creó, en 1929, la Asociación de Mujeres Árabes de Palestina (Arab Women's Association of Palestine, 
AWAP) que tenía un fin político (sin ser reflejo de la vida política masculina) tras la escalada de violencia en la zona que se saldó con la muerte de 133 judíos y al menos 116 árabes (Fleischmann 2000: 17).

Ya en el siglo actual encontramos muchas asociaciones en apoyo a los derechos de las mujeres como, por ejemplo, la Unión de Mujeres Jordanas (Jordanian Women's Union) en Jordania o Network de Mujeres Iraquíes (Iraqi Women Network) En Iraq. También hallamos organizaciones dirigidas por mujeres musulmanas con fines sociales que persiguen la igualdad, la no-discriminación, la justicia y la dignidad como base de toda relación humana, como es el caso de $M u s a w a h^{4}$. Por otro lado, no podemos olvidar el papel principal del activismo feminista en la lucha por la igualdad de género. En Epigto encontramos a Nawal al-Saadawi, la mayor representante del discurso feminista reivindicativo y laico y que fue la primera mujer que introdujo el debate sobre el sexo en las sociedades árabes a través de su obra (Paradela 2015: 25). Así como la vertiente feminista en la figura de la afroamericana convertida al islam Amina Wadud que aboga por un "feminismo islámico":

la verdadera liberación de la mujer (musulmana, por supuesto) no está en el abandono de la propia cultura -entendida, claro, en términos religiosos- y en la adopción de valores extranjeros, sino en la vuelta al islam, en la aplicación de la sharía, una vez, eso sí, depurada de todas las falsas interpretaciones acumuladas tras siglos de exegesis coránica masculina y patriarcal. (Paradela 2015: 25)

\footnotetext{
${ }^{4}$ Esta asociación tiene como fin la igualdad y la justicia en las familias musulmanas, independientemente del país donde se encuentren. Es de importancia mencionarla dado que el Islam es la religión mayoritaria de los países árabes, con excepción del Líbano donde según el informe CIA World Factbook de 2019 tan solo el $57.7 \%$ de la población es musulmana.
} 
En el caso del Líbano fue la escritora Leila Baalbaki quien impulsó el movimiento feminista de este país y, como expone García (2018: 3), "hace de su novela un arma de combate por los derechos de la mujer, que son sus propios derechos". Hay que tener en cuenta una circunstancia básica que ha contribuido al desarrollo de la mujer árabe. Es el hecho de que la mujer ha podido desvincularse gradualmente de la estructura fijada y dominante del clan donde ella jugaba un papel establecido desde su nacimiento. Tal como explica Pappé (2014: 225-273) dos han sido las causas principales que han provocado tal desvinculación y que han reducido el poder de estructura de clan sobre la mujer: por un lado, la migración del campo a la ciudad que produjo un empoderamiento de la mujer resultado de la urbanización; Por otro lado, y más importante, el efecto negativo de la globalización sobre esta estructura afectando a los modos de producción de las familias y, en consecuencia, al concepto de clan. ya que este era una forma de unidad social y productiva que obligaba al matrimonio entre parientes para asegurar de ese modo el mantenimiento de la producción familiar al unirse el capital y los medios de producción. Por tanto, la globalización rompe con estas formas de organización social y jerárquica y debilita el sistema de clanes lo que se traduce en una alteración también de los modos de vinculación social. Ya no es necesario el matrimonio endogámico, la mujer puede elegir con más libertad y, por ende, da un paso adelante en la persecución de su derecho a decidir.

Así pues, la mujer ha logrado la representación en la vida pública de una manera más activa, incluso en puesto de gran responsabilidad como Ministerios. Tal es el caso de Reem bint Ibrahim Al-Hashimy, Ministra de Estado para Cooperación Internacional de Emiratos Árabes Unidos; Dra. Madiha bint Ahmed bin Nasser Al Shaibaniyah, 
Ministra de Educación de Omán; Jamila Moussali, Ministra de Solidaridad, Desarrollo Social, Igualdad y Familia de Marruecos o Hala Zawati, Ministra de Energía y Recursos Minerales de Jordania (Ministerio de Asuntos Exteriores, 2019), y otras tantas embajadoras, alcaldesas y profesoras universitarias.

A pesar de todo esto, la realidad revela que la mujer en el mundo árabe sigue estando en condición de gran desventaja con respecto al hombre. El informe realizado por la Organización para la Cooperación y el Desarrollo Económicos en colaboración con el Centro de la Mujer Árabe para Formación e Investigación (OCDE/CAWTAR 2016) sobre el papel de la mujer de la zona $\mathrm{MENA}^{5}$ en la vida pública pone de manifiesto los éxitos logrados, pero también restricciones y deficiencias de la legalización en muchos de los países de esta zona sobre la igualdad de géneros y derechos de la mujer. No obstante, este informe propone una serie de recomendaciones para avanzar socialmente y en derechos por parte de la mujer, de los cuales resaltamos los que siguen:

- Mejorar el marco institucional y hacer políticas coherentes para el avance en la igualdad de género (7).

- Avanzar en la implementación y coordinación de la igualdad de género y políticas de integración (7).

\footnotetext{
5 El acrónimo MENA significa en inglés Middle East and North Africa (Oriente Medio y Norte de África). Es un término muy ambiguo porque no está definido con exactitud qué países lo conforma. Por lo general se incluye dentro de esta denominación a Algeria, Arabia Saudí, Bahrein, Egipto, EAU (Emiratos Árabes Unidos), Iraq, Jordania, Kuwait, Líbano, Libia, Marruecos, Omán, Palestina (Autoridad), Catar, Somalia, Sudán, Túnez, Yemen, Yibuti y, a veces, países no árabes como, Turquía, Irán e Israel.
} 
- Incrementar la participación femenina en cargos representativos y ejecutivos (15), evitando de tal manera la llamada segregación vertical.

- Mayor representación de la mujer en el poder judicial (16).

- Mejorar la habilidad de la mujer y resaltar su voz y participación en la sociedad civil (17).

- Asegurar la consistencia de la legislación nacional del trabajo con los niveles estándares internacionales (22).

- Mejorar la conciliación entre vida laboral y familiar (24).

- Asegurar la igualdad de género en las relaciones familiares (28).

- Eliminar la violencia contra las mujeres (30).

- Asegurar el mismo acceso a la justicia para hombre y mujeres (32).

En última instancia, un tema aún sin resolver es el de la poligamia que vulnera totalmente el principio de igualdad, excepcionalmente Túnez la abolió en 1956. En otros países árabes ha habido reformas legales en los últimos años con el fin de impedir tal práctica, como que el hombre tenga suficientes recursos para mantener a todas, que notifique a la primera esposa y que exponga razones para justificar tales matrimonios. (OCDE 2017: 81).

2.1. Situación laboral de las mujeres árabes 


\subsubsection{Derechos y sectores}

Contrario a lo que se piensa muchas veces en Occidente, todos los países árabes han adoptado ciertos principios de igualdad en lo referente a la legislación laboral, creando a su vez leyes que protejan a la mujer en ciertas circunstancias laborales. Adscribiéndose a la Convención Para La Eliminación De Toda Forma De Discriminación Contra La Mujer que dictamina en el artículo 11/1 que:

Los Estados Partes adoptarán todas las medidas apropiadas para eliminar la discriminación contra la mujer en la esfera del empleo a fin de asegurar a la mujer, en condiciones de igualdad con los hombres, los mismos derechos, en particular: a) El derecho al trabajo como derecho inalienable de todo ser humano; b) El derecho a las mismas oportunidades de empleo, inclusive a la aplicación de los mismos criterios de selección en cuestiones de empleo y c) El derecho a elegir libremente profesión y empleo, el derecho al ascenso, a la estabilidad en el empleo y a todas las prestaciones y otras condiciones de servicio, y el derecho a la formación profesional y al readiestramiento, incluido el aprendizaje, la formación profesional superior y el adiestramiento periódico. (ONU 1981: 13-143)

En el caso del mundo árabe, la mujer tiende a trabajar en el sector público, ya que ofrece mejores condiciones de trabajo, mejores beneficios como servicios de guarderías, más flexibilidad de horarios y mayor seguridad laboral. Por lo tanto, no es de extrañar que socialmente esté mejor visto que el trabajo en el sector privado y, por este motivo, la OCDE/CAWTAR (2014b: 163) lo indica como un factor determinante a la hora de acceder al mercado laboral. Asímismo, la variación de tipos de trabajos depende mucho de los contextos sociales y culturales del país. Así, según el informe de UNICEF (2011a: 3), en Argelia la mayoría de las mujeres trabajan en el sector del cuidado de la salud, en la educación y en ámbito jurídico, y tan solo el $37 \%$ de mujeres se les 
consideraba activa laboralmente. En Marruecos, por otro lado, las mujeres trabajan principalmente en trabajos mal remunerados asociados a la industria textil, el sector agrícola, trabajadoras domésticas, e, incluso, en trabajos de economía informal (UNICEF 2011c: 4). Con tan solo el 25\% de mujeres activamente laborales en 2017 (World Bank Group 2018: 14). Un caso curioso es el de Catar donde la participación de la mujer de una manera activa en el mercado laboral es muy alta en comparación con otros países de la zona ${ }^{6}$. Con un 58\% de mujeres activas en 2017 (World Bank Group 2018: 12, 14), Catar se sitúa a la cabeza de los países árabes en cuanto a fuerza laboral femenina se refiere. Entre los sectores más destacado están el cuidado de la salud, la educación y labores administrativas (UNICEF 2011e: 4). En contraposición, los trabajos no cualificados suelen recaer en mujeres de otros países, como las trabajadoras domésticas que son en su mayoría de Indonesia (Gardner et al. 2013: 4, 12).

En cuanto a Yemen, la tasa de población activa femenina ha sufrido un gran descenso en los últimos años debido al conflicto bélico, pasando de aproximadamente 20\% en 2011 (UNICEF Yemen 2011f: 4) a tan solo 6\% en 2017 (World Bank Group 2018: 13). En la Autoridad Palestina las mujeres encuentran que sus capacidades y oportunidades están directamente relacionadas con el problema político que sufre la zona. Las restricciones de movimiento en la zona ocupada, los continuos bloqueos en la zona de Gaza y las tensiones políticas entre los grupos Hamás y Fatah han perjudicado el acceso de las mujeres a un trabajo y en muchos casos también a hombres. Por razones económicas, muchas mujeres han realizado trabajos considerados masculinos para los

\footnotetext{
${ }^{6}$ Omán $30 \%$, Kuwait 47\%, Emiratos Árabes Unidos $42 \%$ y Bahrein $44 \%$ aproximadamente ( ver gráfica en World Bank Group 2018: 14)
} 
que no estaban capacitadas, mal pagados y en sectores no protegidos como servicio doméstico y en la agricultura (UNICEF 2011d: 4).

En general, y según el informe del Banco Mundial (World Bank Group 2019: 6), en el año 2018 se estimaba que la fuerza laboral femenina (mayor del 15\%) en el conjunto países de la zona MENA rondaba alrededor del 20\%, tan solo un punto más que en el 2000 que se situaba en el $19 \%$.

2.1.2 Barreras laborales de las mujeres árabes

Con lo expuesto hasta el momento, podemos determinar que la fuerza laboral femenina en los países árabes, y por extensión de la zona MENA, se encuentra condicionadas por una serie de barreras que indica OCDE/CAWTAR (2014b: 167-172) y que especificamos a continuación:

- Barreas legales. Entre las barreras legales se incluye el limitado acceso de las mujeres en algunos países a ciertas profesiones como la de juez o fiscal. En otros, incluso, necesitan el permiso de su guardián (padre, hermano o hijo) para poder obtener un pasaporte, viajar o incluso trabajar fuera de casa. Así como, el sistema de pensiones que favorece considerablemente al hombre (OCDE/CAWTAR 2014b: 167).

- Barreras institucionales. En el plano institucional nos encontramos con que las instituciones sociales siguen considerando al hombre como el principal, y en 
muchos casos, el único capaz de aportar el sustento de la familia. Desde esta perspectiva, todavía existen leyes en algunos países árabes que impiden a las mujeres ser autosuficientes al considerar que solo los hombres pueden protegerlas $\mathrm{y}$, por lo tanto, según la costumbre no deben ni tienen por qué trabajar.

Debemos añadir, además, la falta de políticas para conciliar la vida laboral y familiar y las trabas con las que se encuentran las mujeres para el acceso a servicios gubernamentales. También es importante señalar el papel que juegan los medios de comunicación y el sistema educativo en tanto y cuanto resaltan la tradicional dicotomía de género a favor de los hombres. Los medios de comunicación deben esforzarse en transmitir una imagen más positiva de la mujer como fuerza de trabajo en la vida pública. El sistema educativo, por su parte, debe ser modificado para que permita a la mujer desde los primeros años escolares expresar su decisión en cuanto a su deseo de lo que quiere ser y que no siga el dictamen impuesto por la familia y la sociedad (OCDE/CAWTAR 2014b: 167-169).

- Barreras económicas. En los países árabes el sector público goza de mejor reputación que el sector privado como vimos. Los mejores beneficios, los mejores salarios, la seguridad y la flexibilidad en cuando a turnos de trabajo son determinantes para una mujer a la hora de decidir en qué sector competir, inclinándose la balanza por el público en detrimento del privado donde el número de mujeres trabajadoras suele ser mínimo. Recientemente, sin embargo, los cambios políticos de la zona han repercutido considerablemente en el 
mercado laboral del sector público, reduciendo los puestos de trabajo tanto para mujeres como para hombre. Este hecho dificulta aún más el acceso de la mujer al mundo laboral al tener que competir ahora con el hombre al logro de un puesto de trabajo. Sin embardo, y siendo positivos, esta reducción de puesto de trabajo en el sector público podría aprovecharse y considerarse como una oportunidad para redefinir en primer lugar la confianza del papel de la mujer como trabajadora y motivarla a saltar al sector privado y, en segundo lugar, para cambiar las disposiciones legales de la zona. Por otra parte, otro factor que impide el acceso de muchas mujeres de las mujeres al mercado laboral es la falta de cuidados de sus hijos y las limitadas bajas de maternidad y paternidad (OCDE/CAWTAR 2014b: 169-170).

- Barreras sociales. El papel tradicional de la mujer árabe, asignado por la sociedad, es la casa, el cuidado de las hijas e hijos, personas enfermas y, por supuesto, contribuir al desarrollo adecuado de su familia, la sociedad e, incluso, su propio país. En muchos casos es la familia la que les restringe las capacidades de realización personal inclusive en mujeres altamente cualificadas.

Así pues, una de las barreras sociales más difícil con las que se enfrenta la mujer es la imposición de casarse en edades tempranas. Esto supone un obstáculo para que desarrollen una profesión al no poder conciliar adecuadamente vida laboral y familiar.

Al igual que una barrera legal, se entiende como barrera social la dificultad de elegir dónde vivir, solicitar un pasaporte, viajar fuera del país y en algunos 
países, y casos extremos, ni salir de casa. El llamado “"código de recato” sostiene que la mujer está sujeta al control del hombre como protector (capacidad de trabajo y viaje) ya que es éste quien debe perseverar la reputación familiar y, por lo tanto, ejerce su autoridad sobre la mujer en todas las áreas de su decisión (OCDE/CAWTAR 2014b: 170-171).

- Inestabilidad política de algunos países árabes es otra barrera a la que las mujeres deben enfrentarse. El caso más destacado es el de la Autoridad Palestina donde están expuestas a la vulnerabilidad de las intervenciones militares. Como ya hemos indicado anteriormente, los continuos e interminables controles que hay que pasar para acceder a la zona administrativa desmotivan enormemente a las mujeres a la hora de acceder a un puesto de trabajo. Muchas mujeres palestinas tienen que esperar hasta altas horas para poder volver a sus hogares, lo que provoca un sentimiento de desprotección a la vez que un doble prejuicio: por una parte, la ya mencionada limitación física de las mujeres por los controles, por otra parte, y como consecuencia de todo ello, se intensifica la presión que ejerce sobre ella la estructura social de patriarcado obligándolas a las labores de la casa (OCDE/CAWTAR 2014b: 171-172).

2.2. El caso de la mujer en Jordania como fuerza laboral

Jordania se encuentra situada en Oriente Medio. Limita al norte con Siria, al sur 
con Arabia Saudí y el Mar Rojo, al este con Iraq y al oeste con la Autoridad Nacional Palestina e Israel. El país cuenta con una población de 10.309.000 habitantes (Ministerio de Asuntos Exteriores, Jordania 2019). De mayoría musulmana, tanto la corte civil como la religiosa forman parte del sistema judicial. La ley está basada en el código civil francés, la ley islámica (Sharía) y tiene influencia de tradiciones tribales (UNICEF 2011b: 1). Casi la mitad de la población es femenina con una tasa de participación laboral de tan solo el 14\% en 2017 (World Bank Group 2018: 12) a pesar de tener una tasa 99\% de alfabetización de los jóvenes (UNICEF 2011c: 4). Esto contrasta con el hecho de que Jordania ratificó el 1 de Julio de 1992 la, ya mencionada, Convención para la Eliminación de toda Forma de Discriminación contra la Mujer, pero se reservó dos artículos: el Artículo 9(2): “Los Estados Partes otorgarán a la mujer los mismos derechos que al hombre con respecto a la nacionalidad de sus hijos" (ONU 1981: 73); y el Artículo 16(1c): “Los mismos derechos y responsabilidades durante el matrimonio y con ocasión de su disolución"; (1d): "Los mismos derechos y responsabilidades como progenitores, cualquiera que sea su estado civil, en materias relacionadas con sus hijos; en todos los casos, los intereses de los hijos serán la consideración primordial"; (1g): "Los mismos derechos personales como marido y mujer, entre ellos el derecho a elegir apellido, profesión y ocupación” (ONU 1981: 76).

Jordania se encuadra en los marcos jurídicos y sociales generales mencionados anteriormente (ver epígrafe 2.1.) relativo a la condición legal de la mujer como fuerza laboral de los países de la zona MENA. Entre las características propias de este país en cuestiones de igualdad de género y la posición de las mujeres en el mundo laboral, siguiendo el documento OCDE/CAWTAR (2014b) hay que destacar: 
- La constitución jordana que recoge el principio de igualdad de oportunidades entre hombres y mujeres en cuanto a derechos laborales se refiere (OCDE/CAWTAR 2014b: 175).

- El sistema de pensiones que discrimina a las mujeres jordanas porque tienen la obligación de jubilarse a la edad de 55 frente a los 60 de los hombres. Esto supone una reducción de cinco años en la contribución de las mujeres y una desigualdad frente al hombre que recibe una mejor pensión (OCDE/CAWTAR 2014b: 167).

- En cuanto al horario laboral, la ley en Jordania prohíbe trabajar a las mujeres desde las 8 pm hasta las 6 am, con algunas excepciones. Las horas máximas de trabajo son 10 horas diarias (OCDE/CAWTAR 2014b: 180).

- Con relación a la baja materna, las mujeres jordanas tienen derecho, por ley $\left(n^{\circ} 8\right.$ de 1996), a una baja de maternidad de 11.6 semanas (70 días) en el sector privado frente a 15 semanas (90 días) en el público con sueldo integral. Existe también la posibilidad de pedir baja laboral para cuidar de los hijos por un periodo no superior a dos años con la garantía de conservar su puesto de trabajo. También puede pedir una baja laboral por un periodo máximo de 10 años y con garantía de conservar su puesto de trabajo si debe acompañar a su marido por motivos laborales fuera del reino de Jordania (OCDE/CAWTAR 2014b: 181, 184).

- Respecto al balance entre vida laboral y familiar, la ley jordana ofrece beneficios aportados por el empleador como la baja por enfermedad de un miembro de la familia, medidas especiales para mujeres embarazadas, baja para poder cuidar a 
miembros ancianos de la familia y facilidades para el cuidado de los hijos (OCDE/CAWTAR 2014b: 189).

Además de lo anteriormente mencionado, Jordania está haciendo un gran esfuerzo para poder mejorar la condición de la mujer en la vida laboral y, a su vez, facilitar el acceso de mujeres a puesto de alta responsabilidad. Según el informe de ONU Mujeres (2019a), Jordania se ha comprometido a facilitar políticas que ayuden a las mujeres a ocupar puestos de liderazgos, a participar en la toma de decisiones y también, y muy importante, a transferir medios a la Comisión Nacional Jordana para la Mujer para lograr el empoderamiento de estas a todos los niveles, incluidas refugiadas, así como también el apoyo a mujeres para poder acceder al sistema judicial. En este sentido, y en un esfuerzo hacia la eliminación de la desigualdad de género, Jordania ha aprobado una nueva ley electoral que reserva 15 escaños para las mujeres y en las elecciones parlamentarias del 2016 fueron elegidas 20 mujeres.

Con todo, yo creo que en muchos casos son las propias mujeres jordanas, incluso cualificadas, las que, en parte presionadas por la familia, en parte por decisión propia, deciden no trabajar y quedarse al cuidado de la familia ${ }^{7}$. Sin duda, éste es uno de los mayores problemas a erradicar pues el potencial femenino no es aprovechado

\footnotetext{
7 A modo personal, he de comentar que, durante mis cinco años como lectora de español en la Universidad de Yarmouk, Irbid, al norte de Jordania, y más tarde en Amman, la capital, tuve la gran suerte de conocer de cerca la realidad social jordana a través de mis alumnas. Debido a que el estudio de lenguas modernas era y es aún una especialidad, si razón lógica, relacionada con el sexo femenino, la mayoría de mis alumnos eran mujeres. Muchas de ellas, las de zonas rurales o de clase baja, estudiaban motivadas por salir de casa y conocer a otra gente o/y tener la potestad de elegir un marido acorde con su nivel intelectual, lo que suponía acceder un escalón más alto en su estatus social, o sea, una estrategia para ascender socialmente. Entre ellas, muy pocas, se esforzaban por encontrar un trabajo. Las de zonas urbanas y/o de clase más alta lo hacían por ser la norma en el ambiente social en el que se criaban o por interés en la materia, y a veces, tampoco con interés de trabajar después. Además, algunas ya estaban comprometidas antes de acabar la carrera y casadas uno o dos años después o incluso antes, durante los estudios.
} 
adecuadamente y, por consiguiente, se ve afectada la economía y el avance social del país.

Por último, hay que mencionar el papel de la mujer refugiada en Jordania. El país ha sufrido un incremento no natural de la población debido a las oleadas masivas de refugiados procedentes de los conflictos de países vecinos ${ }^{8}$. La Oficina del Alto Comisionado de las Naciones Unidas para los Refugiados ([ACNUR] 2017: 1) estima que en junio de 2017 había 736.396 refugiados de los cuales 659.593 procedían de Siria, 63.024 de Iraq, 7.916 de Yemen, 3.567 de Sudán y 2.296 de otros países. Centrándonos en los refugiados sirios, el más numeroso (en 2015 el 13,2\% de la población total de Jordania era siria (World Bank Group 2018: 14)), la investigación demuestra que el porcentaje de fuerza laboral de las mujeres sirias tiende a la baja y suelen trabajar menos horas que los hombres y, por lo tanto, salarios más inferiores. A esto hay que añadir la actitud de los trabajadores hacia ellas y la difícil tarea de conciliar vida laboral y familiar (Stave and Hillesund 2015: 88-92). De hecho, el elevado número de hijos que tienen muchas de ellas es un desafío que limita su ingreso en el mercado laboral puesto que se enfrentan a la necesidad de dedicarse al cuidado de los hijos.

Por otro lado, hay una mayor necesidad de que la mujer siria busque trabajo remunerado para poder mantener a sus familias ya que en muchas familias sirias no hay hombre que aporte el dinero a las familias (Errighi and Griesse 2016: 21). Asimismo, el nivel educativo de los refugiados en Jordania suele ser inferior al de su población (tal solo el 3\% de la población femenina del campo de refugiado de Zaatari y el 5\% de fuera

\footnotetext{
${ }^{8}$ En 2013 EE. UU. ocupó Iraq en la llamada segunda guerra del golfo. En 2011 empezó el conflicto sirio y en 2014 el conflicto yemení.
} 
del campo tiene una educación a nivel universitario (ver tabla en Stave y Hillesund 2015: 38)), lo que las condiciona para realizar trabajos no cualificados, en concreto, muchas mujeres trabajan en la agricultura o industria (Razzaz 2017). El sector doméstico en Jordania, sin embargo, está casi exclusivamente reservado a mujeres que no están acompañadas y que viven en la casa donde trabajan. En 2015 la cifra de permisos de trabajos para este sector era 53.882, de los cuales $46 \%$ era para migrantes de Bangladés, 31\% de Filipinas y el resto repartido entre Kenia, Sri Lanka e Indonesia. Curiosamente las mujeres jordanas y las sirias apenas se dedican a este sector, y, si lo hacen, es a tiempo parcial y como externas (Razzaz 2017: 77).

\section{Propuesta de cooperación internacional para el acceso al mundo laboral de la mujer jordana}

Con lo expuesto en los epígrafes anteriores, en las siguientes páginas ofrecemos nuestra propuesta para empoderar a la mujer en Jordania. Conviene comenzar indicando que las ONGs jordanas están jugando un papel primordial en los cambios sociales que sufre el país y prueba de ello son los numerosos proyectos que tienen en toda Jordania. El objetivo principal de estas ONGs locales es el desarrollo humano del país, destacando la importancia de la mujer como fuerza laboral y ayudando a su empoderamiento mediante el conocimiento de sus propias capacidades. Entre estas ONGs podemos destacar el Fondo jordano Hachemita para el Desarrollo Humano (Jordanian Hashemite Fund for Human Development (JHUD)), la Fundación Río 
Jordán (Jordan River Foundation), Fundación Noor al-Hussein (Noor al-Hussein Foundation), el Foro Nacional Jordana para Mujeres (Jordanian National Forum for Women) y la Comisión Nacional Jordana de la Mujer (Jordanian National Commission for Women).

La propuesta que se presenta en este capítulo persigue los mismos objetivos que estas ONGs y, se enmarca, por lo tanto, dentro de un programa de desarrollo humano que busca el empoderamiento económico de la mujer jordana con menos posibilidades a través de pequeños negocios familiares. Se pretende de esta manera promover la difícil labor de la igualdad de oportunidad de género, fomentar en las mujeres el espíritu empresarial, promover su participación dentro de la comunidad a la que pertenecen y romper con la tradición de postergación e inequidad.

Habida cuenta de los impedimentos sociales y legales ya expuestos, es necesario definir qué tipo de trabajo son viables para el aprovechamiento de las capacidades de las mujeres, proporcionándoles las posibilidades y recursos para trabajar por cuenta propia y acabar de este modo con la exclusión sociolaboral. Con ello podrá contribuir a la carga económica familiar y a la nacional y, en definitiva, incrementar sus beneficios sociales y económicos.

\subsection{Microempresas familiares en Jordania}

La propuesta de las microempresas familiares dirigidas por mujeres está planteada inicialmente para ser realizada dentro de un proyecto de cooperación internacional que 
ayude a empoderar a las mujeres que bien por presiones familiares o sociales no pueden acceder a un trabajo en sectores para los que es necesario alejarse de su lugar de residencia, o bien que el trabajo que quieren hacer no se considere "moralmente aceptados" por el entorno donde viven.

Por lo tanto, las ventajas principales de las que se aprovecha la mujer son: el horario flexible que tiene y la cercanía del entorno donde se pueden llevar a cabo. Estas dos ventajas van a permitir conciliar más fácilmente el mundo laboral y familiar.

Las propuestas de microempresas son: confección, artesanía, educación: academias en casa, cuidado de niños de mujeres trabajadoras y cooperativa de pan y dulces tradicionales.

\subsubsection{Confección}

El negocio de la confección ofrece a la mujer la posibilidad de trabajar desde casa o en un taller cerca de su lugar de residencia. De esta manera se pretende que la mujer contribuya a los ingresos de la familia vendiendo su propia ropa o haciendo arreglos de ropa.

Por regla general son mujeres con nivel educativo no muy alto, o bien residen en zonas rurales donde resulta más difícil, por causas familiares, sociales o tan simples como falta de transportes, desplazarse hasta un puesto de trabajo en zona urbana. Los recursos que necesitará cada mujer para poder crear la microempresa en el sector textil 
son los siguientes: taller de costura, máquinas de costura, telas, mesa de costura, accesorio de costura, ordenador y acceso a internet y ayuda para aprender a publicitarse. Antes de acceder a las ayudas, las mujeres deberán realizar un curso de formación básica sobre corte y confección destinado a crear mano calificada femenina en la cadena de producción para que pueda integrarse posteriormente en el mercado textil.

\subsubsection{Artesanía}

Este tipo de actividad sigue la misma línea que la de confección, aunque con las características propias de la artesanía.

Este trabajo está más encaminado a mujeres que viven en zonas turísticas donde es más fácil vender los productos elaborados. Debido a que ya existen mujeres dedicadas a esta labor (ver, por ejemplo, El Centro de Artesanía de la cuidad de Madaba y, Wadi Musa, cerca de Petra donde hay una fábrica de pulseras y brazaletes) lo más adecuado es crear estrategias para seguir motivando a las mujeres que ya trabajan en artesanía e incentivar a otras a realizar este tipo de trabajo. Un punto importante que hay que destacar es que sean las propias mujeres las que dirijan el negocio.

Los recursos que necesitará cada mujer, o en este caso grupo de mujeres, para poder crear la miniempresa en el sector artesanal son: financiación para mejorar el negocio ya establecido y ampliarlo integrando a otras mujeres, herramientas básicas 
para realizar los productos artesanos que realizan, ordenador y acceso a internet y ayuda para aprender a publicitarse.

Una iniciativa parecida la puso en marcha ONU Mujeres (2019b) para empoderar económicamente a la mujer y fomentar la capacidad empresarial y el futuro de campesinas y mujeres refugiada. Se realizó en el Líbano con subvención procedente del Fondo para la Igualdad de Género de Naciones Unidas y de la que fue beneficiaria la Asociación Amel Internacional (Amel Association International). El proyecto fue todo un éxito y actualmente hay más de 300 productoras entre refugiadas y campesinas libanesas que venden jabones, velas, accesorios y artesanías directamente al público.

\subsubsection{Educación: academias en casa}

Como se señaló en el apartado anterior, el índice de mujeres que acceden a la universidad es muy alto comparado con el índice de mujeres incorporadas al mundo laboral. Esto es debido a que muchas de ellas tras acabar, o incluso antes de acabar sus estudios, deciden formar una familia bloqueando así posteriormente cualquier esfuerzo de acceder a un trabajo.

Dada la capacidad intelectual de estas mujeres se pretende que aprovechen tal potencial intelectual y lo transmitan a las generaciones más jóvenes en edades escolares. Esto supone un doble aprovechamiento pues no solo empodera económicamente a la mujer, sino que además ayuda a elevar el nivel educativo de los niños y jóvenes. 
Los recursos que necesitará cada mujer para crear una microempresa en el sector educativo son las siguientes: sala para dar clase, material escolar, mesas y sillas para los alumnos, encerado normal o electrónico, ordenador y acceso a internet y ayuda para aprender a publicitarse.

\subsubsection{Cuidado de niños de mujeres trabajadoras}

Esta propuesta va dirigida a mujeres con nivel de estudios medio/alto que no trabajan. La idea es que en su propio espacio acojan a un grupo reducido de niños, máximo 6 (incluido los suyos propios) y los cuiden durante las horas que sus madres trabajan en otros sectores ${ }^{9}$. Debido a que implica trabajar con niños, se debe exigir a la cuidadora cumplir con cierto tipo de requisitos, entre ellos psicológicos y sanitarios.

Para empezar, hay que hace un examen y reconocimiento a la cuidadora para saber si está capacitada para tal labor. De igual manera se debe inspeccionar si el lugar donde van a estar los niños está en condiciones tanto de limpieza como de espacio para acogerlos. En un principio este trabajo está pensado para que se realice en casa de la cuidadora pues se da por hecho que ella también tiene hijos a los que cuidar. Si no es posible, sería recomendable habilitar un lugar alquilado con las normas de seguridad y sanitarias que debe cumplir este tipo de habitáculo para tales efectos.

\footnotetext{
${ }^{9}$ Este tipo de cuidado de niños está representado en Alemania en la figura de las Tagesmütter 'madres de día', está legalmente regulado y su servicio se solicita a través de la Oficina de juventud de su distrito o localidad. Es indudable que la realidad social de Jordania no es la misma que la Alemania y de que los recursos económicos y sociales distan mucho de equipararse, pero a mi juicio la idea de base puede ser aplicable igualmente en los dos países.
} 
Antes de empezar tal labor es necesario que la mujer realice un curso sobre primeros auxilios para niños y conocimientos básicos en educación en edad preescolar. El horario es el que corresponde a una jornada laboral normal en Jordania, con un margen de media hora antes y después para que las madres y los padres puedan dejar y recoger a los niños y niñas. Los recursos que necesitará la mujer para crear las microempresas de cuidadora de niños y niñas son los siguientes: un local amplio donde los niños y las niñas puedan jugar y dormir en caso de que no sea viable en la propia casa, juguetes infantiles hasta edad escolar, material escolar de preescolar para niños y niñas en edad más avanzada y camas pequeñas y cuna.

\subsubsection{Cooperativa de pan y dulces tradicionales}

Este tipo de propuesta tiene como fin que un grupo de mujeres cree una cooperativa para fabricar pan de modo tradicional y dulces. En Territorios Palestinos Ocupados, ONU Mujeres (2019c), a través del Fondo de Igualdad de Género, realizó un programa con el fin de apoyar las cooperativas existentes que están dirigidas por mujeres y a la vez abrir algunas nuevas. El programa fue un éxito absoluto ya que consiguió que muchas mujeres tuvieran nuevas capacidades, una nueva fuente de ingresos mensual y confianza en sí mismas. Por otro lado, estas cooperativas han comenzado a crear coaliciones de defensa, trabajando juntamente con los sindicatos y las organizaciones de la sociedad civil para, entre otras cosas, lograr una mejor protección social para las trabajadoras informales. 
En el caso de esta propuesta, los recursos que necesitará cada mujer o grupo de mujeres para crear una microempresa de cooperativa de pan y dulce son los siguientes: sala grande, horno de pan, utensilios para trabajar el pan y su empaquetado, mesas de trabajo y mostradores, uniformes y medidas sanitarias para este trabajo y financiación para comprar la materia prima.

\subsection{Cursos y talleres de formación}

Para que la mujer siga constantemente trabajando y las propuestas no se agoten en ellas mismas, se recomienda formarla para que entienda la importancia del trabajo que realiza, lo que aporta a la sociedad, así como que sepa distribuir ecuánimemente las horas de trabajo, las ganancias y pérdidas y que aprenda a usar los recursos y los conocimientos adquiridos. Esto se llevará a cabo a través de cursos y talleres que se celebrarán periódicamente y que tendrán el contenido que se indica a continuación:

- Creación de foros y espacios de encuentro que ayuden a intercambiar experiencias y sirva de aprendizaje en conjunto. Según el sector y la zona o ciudad, se debe crear una red que agrupe a un mínimo de 10 mujeres que estarán en permanente contacto y que se reunirán una vez al mes por turno en casa o taller de una de ellas,

- Talleres de autoayuda para apoyar a las mujeres a tener confianza y fortalecerse con la difícil tarea de la actividad empresarial que va a emprender, 
- Talleres que ayuden a crear estrategias para aprender a iniciar, planificar y gestionar un negocio. De esta forma se aumenta su autonomía y con el trabajo también aumentan los ingresos de la familia, y, por último,

- Cursos específicos de motivación y formación destinados a mujeres sobre el uso y aplicación de las nuevas tecnologías y su utilidad para promocionar su empleo.

Tabla I. Estructura de los cursos y talleres

\section{ESTRUCTURA CURSOS Y TALLERES}

\begin{tabular}{|c|c|c|c|}
\hline Asistentes & Periodicidad & Horario & Lugar \\
\hline $\begin{array}{l}\text { 20/25 mujeres por clase* } \\
\text { (* los cursos se realizarán en dos } \\
\text { días debido al alto número de } \\
\text { asistentes, ver tabla Modo de } \\
\text { financiación del Proyecto) }\end{array}$ & $\begin{array}{l}6 \text { sesiones* } \\
(* \text { Cada } 4 \text { meses } \\
\text { durante } 24 \text { meses })\end{array}$ & $9 \mathrm{am}-1 \mathrm{pm}$ & $\begin{array}{l}\text { Sala de la Universidad de } \\
\text { la ciudad más cercana }\end{array}$ \\
\hline
\end{tabular}

\subsection{Duración y fases del proyecto}

El proyecto está pensado para realizarse en 6 años y en 3 fases, cada una corresponde a dos años. En cada fase se elegirá una zona de Jordania con una ciudad principal como centro para realizar los cursos de formación y el lugar donde se realicen los espacios de encuentros. Las zonas están repartidas entre: zona centro (fase 1), provincias de Ammán, Balqa y Mádaba; zona norte (fase 2) provincias de Irbid, Zarqa, Mafraq, Jerash y Ajlun y zona sur (fase 3) con las provincias de Tafila, Karak, Maan y Aqaba. Debido a que la distancia entre los centros de trabajos de la zona centro y sur es 
muy grande, hay tres ciudades, Tafila, Karak y Maan que se quedan aisladas, lejos tanto de la zona centro como de la del sur. Se recomienda en ese caso que la fase 3 , correspondiente a la zona sur, se divida en tres pequeñas zonas de acción: Karak-Tafila, Maan y Aqaba.

En el caso de la propuesta de la Artesanía, no es equitativo el número de mujeres que se pueden beneficiar en cada fase al existir el factor turístico más intenso según la zona. En la fase 2 (zona norte) tan solo dos pequeñas ciudades, Jerash y Ajlun, podrían disfrutar de esta ayuda, y no es seguro que haya muchas mujeres que quieran emprender en este sector. En el caso de la fase 1 (zona centro) y la fase 3 (zona sur) el número de posibles emprendedoras artesanales aumenta, pero se compensa con menos densidad de población en la fase 3 (zona sur).

Tabla II. Esquema del Proyecto

\section{ESQUEMA DEL PROYECTO}

\begin{tabular}{|c|c|c|c|}
\hline Fase & Duración & Región & Propuestas \\
\hline 1 & $\begin{array}{l}2 \text { años } \\
\left(1^{\circ}-2^{\circ} \text { año }\right)\end{array}$ & Zona Centro. Prrovincias: Ammán, Balqa y Mádaba & todas \\
\hline 2 & $\begin{array}{l}2 \text { años } \\
\left(3^{\circ}-4^{\circ} \text { año }\right)\end{array}$ & $\begin{array}{l}\text { Zona Norte. Provincias: Irbid, Zarqa, Mafraq, Jerash y } \\
\text { Ajlun }\end{array}$ & todas \\
\hline 3 & $\begin{array}{l}2 \text { años } \\
\left(5^{\circ}-6^{\circ} \text { año }\right)\end{array}$ & Zona Sur. Provincias: Karak-Tafila, Maan y Aqaba & todas \\
\hline
\end{tabular}

\section{Fuente propia}

Mapa 1. Distribución de las fases y zonas del proyecto en Jordania. Fuente propia 


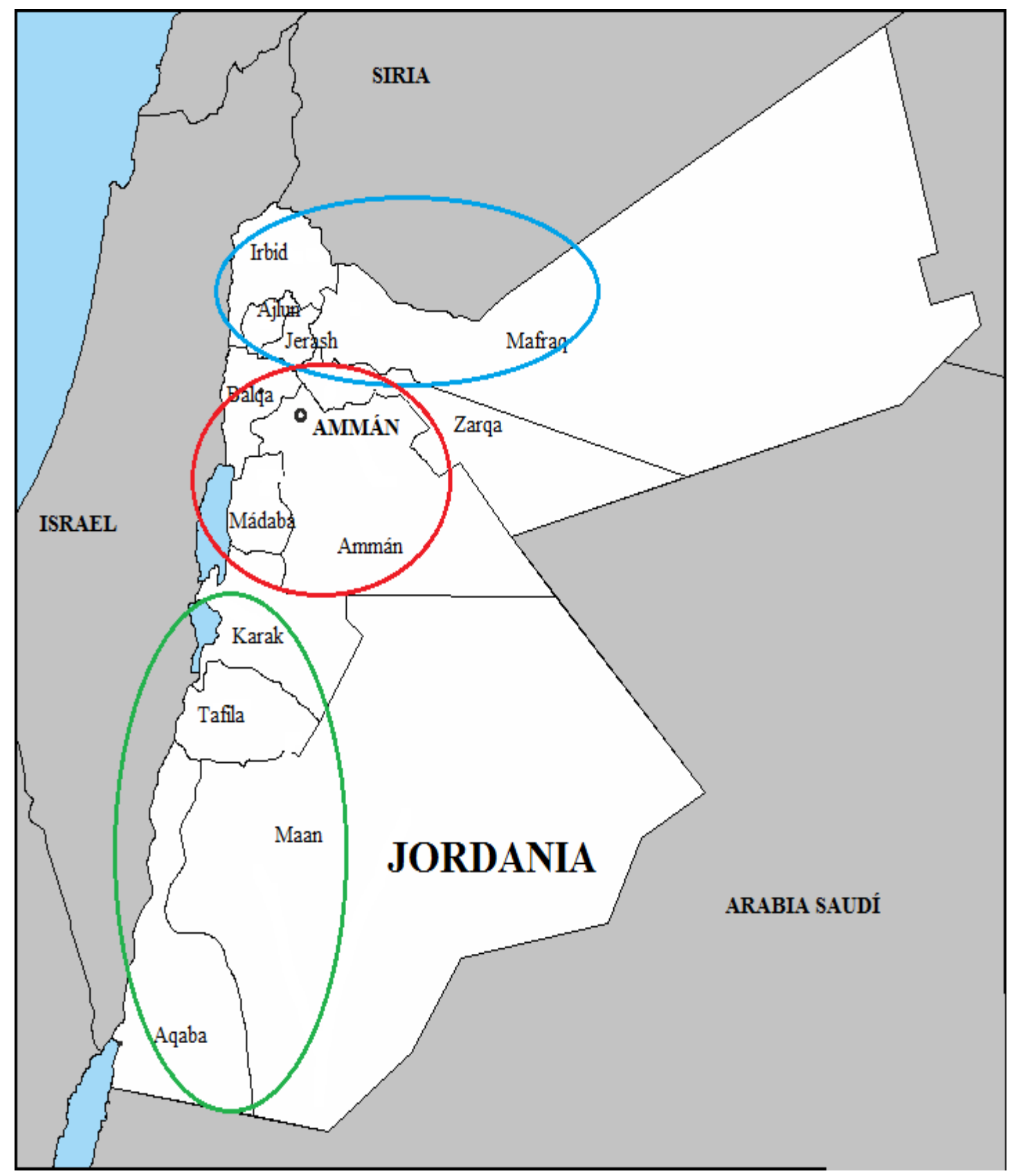

Fase 1. Zona centro

Fuente propia

Fase 2. Zona norte

Fase 3. Zona sur

3.4. Formas de promoción / publicidad 
La publicidad que se haga del proyecto es de suma importancia para asegurarse que el anuncio de éste llega al máximo número de mujeres.

A continuación, se enuncia las maneras y sitios de difusión de la noticia:

- Medios de comunicación: las radios locales, prensa y televisión local,

- Folletos repartidos en lugares de concurrencia femenina: mercados, mezquitas y universidades. En las universidades hay muchas jóvenes que una vez enteradas de la noticia la pueden transmitir a familiares y vecinas que reúnan las condiciones y quieran montar su propia miniempresa. En este apartado se pueden también incluir las escuelas y guarderías públicas,

- Redes sociales. Tal vez sea este uno de los mejores métodos de difundir la noticia del proyecto ya que muchas mujeres jordanas tienen acceso a las diferentes redes sociales,

- Pero sin duda alguna, el mejor de los medios de divulgación del futuro proyecto es a través del boca a boca entre mujeres. En Jordania existe una gran comunicación entre familiares, vecinos y amigos, en la mayoría de los casos diaria y sin previo aviso. Esto hace que cualquier novedad se expanda rápidamente. Esta será la forma más eficaz de hacer llegar la propuesta del proyecto al mayor número de mujeres.

En cuanto a las vías institucionales para dar a conocer el proyecto, se nombran: 
- Presentar el proyecto a los gobiernos locales para que éstos se encarguen de difundirlo a través de tablones de anuncios, boletines, folletos, clips de videos, fotos etc.,

- Convocar charlas y coloquios en instituciones públicas,

- Campañas de promoción,

- Crear consultorías dirigidas por mujeres para que asesoren a las mujeres que deseen, $\mathrm{y}$

- Anuncio de los Premios al emprendimiento. Estos premios persiguen estimular y motivar a las emprendedoras.

3.5. Fundraising y participación de los diferentes actores

\subsubsection{Fundraising: Microcréditos}

Para que las mujeres puedan crear pequeñas y medianas empresas o bien quieran ampliar y mejorar sus negocios se propone un proyecto de cooperación norte-sur entre una Organización Internacional y el Ministerio de Desarrollo Social de Jordania. 
El instrumento financiero será el de microcréditos ${ }^{10}$ que concederá el Ministerio de Desarrollo Social de Jordania, a través de una subvención de una Organización Internacional $^{11}$.

En el caso de los negocios familiares de las mujeres jordanas y conforme a los recursos necesarios que necesitan, lo más adecuado es conceder microcréditos por una cuantía máxima de 1600 JD (dinares jordanos. Ver cuadro página siguiente para las cuantías). El plazo de amortización será de 2 años y sin tipo de interés.

La idea es que con el dinero devuelto de la fase 1 (zona centro) se financie a las mujeres de la fase 2 (zona norte) y con el dinero devuelto de la fase 2 se financie a las mujeres de la fase 3 (zona sur). De ahí que una vez cumplido el plazo de pago y el rembolso total de la cuantía, las mini empresarias podrán solicitar otro microcrédito a la misma institución pasados los 6 años que dura el proyecto.

La financiación de la subvención se repartirá durante las 3 fases siguiendo el siguiente esquema y teniendo en cuenta la subida de los precios y vida durante los 6 años.

\footnotetext{
${ }^{10}$ Comparar el programa Fondo Mujer de El Salvador, un programa de créditos blandos para facilitar el acceso a de la mujer al mundo laboral e incentivar a las que ya tienen negocios a que los potencien. Los socios colaboradores de este programa son Naciones Unidas Mujeres con cooperación con otros socios, La Agencia Italiana de Cooperación para el Desarrollo (AICS), la Secretaría de Inclusión Social (SIS)Ciudad Mujer y el Banco de Desarrollo de El Salvador (BANDESAL). (Ciudad Mujer. Secretaría de Inclusión Social, 2017).

${ }^{11}$ Creemos oportuno que esta Organización Internacional podría ser la ONU y podría financiar el proyecto a través del Fondo para la Igualdad de Género de Naciones Unidas Mujeres y el Comité Nacional alemán de la misma organización. Este fondo concede subvenciones para empoderar económica y políticamente a la mujer y ofrece apoyo y financiero a las iniciativas de emprendimiento, con especial atención a mujeres en situación de marginación. Ver más en: ONU MUJERES (2019d). Un ejemplo lo tenemos en este mismo capítulo en el apartado de microempresas, artesanía.
} 
Tabla III. Modo de financiación del proyecto

\begin{tabular}{|c|c|c|c|c|}
\hline & O DE $\mathrm{F}$ & CIÓN DEL PROY & TO & \\
\hline Fase & Años & Núm. de mujeres & $\begin{array}{l}\text { Cuantía máxima } \\
\text { por mujer }\end{array}$ & Total en JD \\
\hline 1 (zona centro) & $1^{\circ}-2^{\circ}$ & 300 & $1000 \mathrm{JD}$ & $300000 \mathrm{JD}$ \\
\hline 2 (zona norte) & $3^{\circ}-4^{\circ}$ & 200 & $1300 \mathrm{JD}$ & $260000 \mathrm{JD}$ \\
\hline 3 (zona sur) & $5^{\circ}-6^{\circ}$ & 100 & $1600 \mathrm{JD}$ & $160000 \mathrm{JD}$ \\
\hline
\end{tabular}

Fuente propia

El número de mujeres que se beneficiará del microcrédito se va reduciendo a lo largo del proyecto por dos motivos principales: por un lado y el principal, porque las zonas que corresponden a cada fase no contienen el mismo número de habitantes. La zona centro (fase 1) está mucho más poblada que la zona sur (fase 3), con lo que es más justo el reparto de las ayudas y además hay más mujeres emprendedoras por una cuestión numérica. Por otro lado, y como ya hemos comentado, hay que tener en cuenta la subida de los costes de la vida a lo largo de los 6 años que dura el proyecto. Por lo tanto, el cálculo estimado es 300000 JD serán para la fase 1; 260000 JD para la fase 2 y $160000 \mathrm{JD}$ para la fase $3^{12}$.

\subsubsection{Actores de la cooperación}

Donante: Organización Internacional.

\footnotetext{
${ }^{12}$ Las cifras son aproximadas. En la fase 3 (zona sur) la financiación se reduce considerablemente puesto que hay que considerar los gastos inesperados a lo largo de los cuatro primeros años del proyecto.
} 
El socio donante será una Organización Internacional (ver 3.5.1.) que aportará la cantidad para llevar a cabo el proyecto y colabore estrechamente con el beneficiario mediante el conocido "trabajo de socios". Ofrece subvenciones para mujeres emprendedoras jordanas con un aporte financiero para 6 años.

Beneficiario y ejecutor: Ministerio de Desarrollo Social de Jordania.

El socio beneficiario y ejecutor del proyecto será el Ministerio de Desarrollo Social de Jordania. Está presidido por una mujer, la ministra H.E. Hala Lattouf, que tiene una gran trayectoria en la lucha por los derechos de la mujer en Jordania. La misión del socio ejecutor será garantizar la implementación del programa satisfaciendo las condiciones financieras, operativas y técnicas esenciales del proyecto mediante los siguientes medios:

a. Anunciar la ayuda y hacerla llegar al mayor número de mujeres.

b. Asesorar a las mujeres través de consultorías durante los 4 meses que dura la convocatoria abierta de la solicitud. En estas consultorías se explicarán:

- las propuestas para las que pueden solicitar el proyecto,

- cómo hacer un proyecto. Facilitar un modelo estándar de cómo presentar y elaborar un plan de empresa,

- simplificar el aparato burocrático con menos exigencias de documentos para aligerar así el proceso e incentivar a las solicitantes,

- en qué consisten los microcréditos, y 
- plan del proyecto, cursos y talleres obligatorios.

c. También se encargará de hacer llegar los recursos a las futuras microempresarias jordanas, repartiendo la ayuda equitativamente y conforme a igualdad y necesidad de cada caso y no a beneficio personal. Se procurará, en la medida de lo posible que no haya trato de favor hacia un sector o grupo de mujeres.

d. Financiar y organizar mediante profesionales expertos jordanos de contraparte el programa de formación, asesoramiento técnico y financiero y talleres de autoayuda e informática que se realizarán al principio del proyecto y, cada cuatro meses, durante los dos años que dure cada fase.

e. Monitoreo y control del proyecto. El Ministerio contratará a un par de personas expertas para verificar el correcto funcionamiento del proyecto y el destino seguro de los fondos para miniempresas y no para disfrute de las solicitantes. Estos expertos deberán además proporcional información fundamentada y fiable sobre el avance del proyecto.

f. Organizar el evento de Premios al emprendimiento. Estos se entregarán al final de cada fase en una ceremonia en la que no solo estarán invitadas personas importantes de la vida pública jordana, sino también representaciones diplomáticas y de las cámaras de comercios de otros países en Jordania. Dos son los objetivos que se pretenden con estos premios: por una parte, motivar a las mujeres a realizar su labor que va a ser reconocida al final del proyecto y, por 
otra, dar a conocer el potencial de la propuesta de artesanía para abrir las puertas del mercado local a un mercado más internacional.

Beneficiario directo: el colectivo de mujeres que reciben la ayuda.

En cuanto al beneficiario directo, éste será el colectivo de mujeres que van a disfrutar de la ayuda que les proporcione el microcrédito para poder poner en marcha el negocio pensado.

Respecto a las aportaciones económicas que cada actor debe hacer, la Organización Internacional ofrecería una cuantía en USD correspondiente a 300.000 JD (dinares jordanos) en el momento del comienzo del proyecto y teniendo en cuenta el tipo de cambio. Por otro lado, el socio beneficiario, el Ministerio de Desarrollo Social de Jordania, se comprometerá a pagar el servicio de los recursos humanos (personal consultoría, profesionales para cursos y talleres y personal para monitoreo), el material para cursos y talleres y la publicidad que se haga del proyecto:

- Consultoría. En primer lugar, hay que disponer de personal de consultoría en cada ciudad importante de cada fase. Este grupo trabajará durante un periodo máximo de 4 meses, justo desde la fecha de publicación de la ayuda hasta la fecha de cierre de solicitudes. Así pues, se dispondrá de 12 personas, preferiblemente mujeres, repartidas de la siguiente manera: Fase 1, contratación de 4 personas (2 para Ammán, 1 para Mádaba y 1 para Balqa); Fase 2, contratación de 5 personas (1 para Irbid, 1 para Zarqa, 1 para Mafraq, 1 para Jerash y 1 para Ajlun) y Fase 3, contratación de 3 personas (1 para Tafila-Karak, 
1 para Maan y 1 para Aqaba). El importe será de 200 JD mensuales por persona. El importe total de la consultoría en los 4 meses es de 9.600 JD.

- Profesionales de los cursos. El socio beneficiario deberá pagar a los profesionales que van a realizar los cursos y talleres. Serán diez personas que se desplazarán al lugar donde se realizarán las sesiones en cada fase. En la fase 1 (zona centro) y fase 2 (zona centro), el importe por sesión será de 80 JD (incluido transporte) por personas, o sea, 800 JD (10 personal por sesión) por 12 sesiones (6 por cada fase) hace un total de 9.600 JD. Se suma a esta cifra el importe a pagar a los profesionales de la fase 3. Como algunos (si no todos) profesionales de esta fase 3 (zona sur) residen muy lejos de las ciudades donde se realizarán esta fase, el importe a estos profesionales será de 100 JD por sesión, además serán solo 6 profesionales ya que el número de mujeres futuras empresarias es menor. Con lo cual será 600 JD (6 personas por sesión) por 6 sesiones hacen un total de $3.600 \mathrm{JD}$. El importe total del personal en los 6 años de proyecto es de 13.200 JD.

- Material y publicidad. Hay que añadir el material de los cursos y talleres, así como la publicidad que hagan de los cursos. El importe de este material es del $1.000 \mathrm{JD}$.

- Monitoreo. Por último, corre a cuenta del socio jordano el pago del monitoreo y control del proyecto. Se estipula que el gasto para esta gestión será de 6.000 JD en los 6 años del proyecto total. 
Tabla IV. Aportación económica de los actores

\section{APORTACIÓN ECONÓMICA DE LOS ACTORES}

\begin{tabular}{|c|c|c|c|c|c|}
\hline \multirow[t]{2}{*}{$\begin{array}{l}\text { Organización } \\
\text { (donante) }\end{array}$} & \multicolumn{5}{|c|}{$\begin{array}{l}\text { Ministerio de Desarrollo Social de Jordania (beneficiario y ejecutor) } \\
\begin{array}{l}\text { Consultoría: Personal de asesoramiento para ayudar a solicitar la ayuda. Máximo } \\
4 \text { meses. }\end{array}\end{array}$} \\
\hline & Fase & $\begin{array}{l}\text { Núm. } \\
\text { personal }\end{array}$ & \multicolumn{2}{|c|}{$\begin{array}{l}\text { Importe mensual por } \\
\text { persona }\end{array}$} & Total mensual \\
\hline & Fase 1 & 4 & \multicolumn{2}{|c|}{$200 \mathrm{JD}$} & $800 \mathrm{JD}$ \\
\hline & Fase 2 & 5 & \multicolumn{2}{|c|}{$200 \mathrm{JD}$} & $1.000 \mathrm{JD}$ \\
\hline & Fase 3 & 3 & \multicolumn{2}{|c|}{$200 \mathrm{JD}$} & $600 \mathrm{JD}$ \\
\hline & & & \multicolumn{3}{|c|}{$2400 \mathrm{JD} \times 4$ meses $=9.600 \mathrm{JD}$} \\
\hline & \multicolumn{5}{|c|}{ Profesionales para impartir los cursos y talleres. } \\
\hline & Fase & $\begin{array}{l}\text { Núm. } \\
\text { personal }\end{array}$ & $\begin{array}{l}\text { Importe } \\
\text { sesión- } \\
\text { persona }\end{array}$ & $\begin{array}{l}\text { Núm. } \\
\text { sesión }\end{array}$ & Total \\
\hline & Fase 1 & 10 & $80 \mathrm{JD}$ & 6 & $4.800 \mathrm{JD}$ \\
\hline & Fase 2 & 10 & $80 \mathrm{JD}$ & 6 & $4.800 \mathrm{JD}$ \\
\hline & Fase 3 & 6 & $100 \mathrm{JD}$ & 6 & $3.600 \mathrm{JD}$ \\
\hline & \multicolumn{5}{|r|}{13.20} \\
\hline & \multicolumn{3}{|c|}{$\begin{array}{l}\text { Monitoreo } \\
\text { Material de los cursos y publicidad } \\
\text { Evento Premios al Emprendimiento } \\
\text { Aporte total del Ministerio: }\end{array}$} & \multicolumn{2}{|r|}{$\begin{array}{r}6.000 \mathrm{JD} \\
1.000 \mathrm{JD} \\
500 \mathrm{JD} \\
\mathbf{3 0 . 3 0 0} \mathrm{JD}\end{array}$} \\
\hline
\end{tabular}

\section{Conclusiones}

Como hemos visto, el acceso de la mujer árabe, y más concretamente de la mujer en Jordania, a un puesto laboral no es tarea fácil. En este sentido, tanto la aplicación de la ley islámica (sharía) como las normas sociales impiden el desarrollo laboral y social de muchas mujeres incluso altamente cualificadas.

Para paliar esta situación problemática de desigualdad, se ha expuesto una propuesta de cooperación entre una Organización Internacional, como socio donante del 
proyecto, y el Ministerio de Desarrollo Social de Jordania, como socio ejecutor, con el fin de empoderar económicamente a un grupo de mujeres que las ayude a crear sus negocios y motivándolas a ampliarlos.

En este sentido, el proyecto está pensado para facilitar la creación de las siguientes microempresas: confección, artesanía, educación: academias en casa, cuidado de niños de mujeres trabajadoras, cooperativa de pan y dulces tradicionales. Está dirigido a un grupo de 480 mujeres con una duración de 6 años y financiado a través de microcréditos con una cuantía máxima de 1.900 JD por solicitante según la fase.

Consideramos por nuestra experiencia en este país, que de esta forma se incentiva a las mujeres a tomar las riendas de sus propios negocios aumentando tanto su bienestar económico y social como el de sus familias y, en consecuencia, empodera a la mujer mejorando su autoestima. En definitiva, se da un paso más en la difícil tarea de lograr la igualdad de derechos laborales entre hombres y mujeres y, por ende, en el contexto para su aplicación que ha sido Jordania.

\section{Referencias bibliográficas}

ACNUR. Oficina del Alto Comisionado de las Naciones Unidas para los Refugiados. 2017. UNHCR Agency. The UN Refugee. Jordan. Factsheet June 2017. https://reliefweb.int/sites/reliefweb.int/files/resources/Jordan\%20Fact\%20Sheet\% 20June\%202017-\%20FINAL.pdf [09/12/2019].

Errighi, Lorenza and Jörn Griesse. 2016. "The Syrian Refugee Crisis: Labour Market Implications in Jordan and Lebanon”. European Economy Discussion. Paper 029. Luxembourg: European Union. 
Fleischmann, Ellen L. 2000. "The Emergence of the Palestinian Women's Movement, 1929-39”. Journal of Palestine Studies, 29.3 (2000): 16-32.

García Lafuente, María Isabel. 2018. "Leila Baalbaki: la rebelión en voz de mujer". Tonos digital: Revista de estudios filológicos, 35 (2018), 1-17. http://www.tonosdigital.es/ojs/index.php/tonos/article/view/1998/1008 [09/12/ 2019].

Gardner, Andrew et al. 2013. "A Portrait of Low-Income Migrants in Contemporary Qatar". Journal of Arabian Studies, 3.1 (2013): 1-17.

OCDE-Organización para la Cooperación y el Desarrollo Económicos. 2017. Women's Economic Empowerment in Selected MENA Countries. The Impact of Legal Frameworks in Algeria, Egypt, Jordan, Libya, Morocco and Tunisia. https://dx.doi.org/10.1787/9789264279322-en [01/12/2019].

OCDE/CAWTAR-Centro de la Mujer Árabe para Formación e Investigación (CAWTAR por sus siglas en inglés). 2014a. "Towards gender-responsive laws and policies in the MENA region". Women in Public Life Gender, Law and Policy in the Middle East and North Africa. Paris: OECD Publishing. 41-58. DOI: http://dx.doi.org/10.1787/9789264224636-en [09/12/2019].

2014b. "Female Participation in the Labour Force across the MENA Region", in Women in Public Life: Gender, Law and Policy in the Middle East and North Africa. Paris: OECD Publishing. 157-210. DOI: http://dx.doi.org/10.1787/9789264224636-10-en [09/12/2019].

\section{Middle}

2016. Report on Women in Public Life: Gender, Law and Policy in the

East and North Africa. MENA-OECD Governance Programme. En colaboración con Centro de la Mujer Árabe para Formación e Investigación (CAWTAR por sus siglas en inglés). Paris: OECD Publishing.

ONU. 1981. Convention on the Elimination of All Forms of Discrimination against Women (CEDAW). Adopted by the General Assembly of the United Nations on 18 December 1979, Treaty Series, 1249, $\mathrm{n}^{\circ} 20378$ (1981): 13-143, (versión en español pp. 70-79). https://treaties.un.org/doc/Publication/UNTS/Volume \%201249/v1249.pdf [09/12/2019].

ONU Mujeres-(Entidad de la ONU para la Igualdad de Género y el Empoderamiento de la Mujer). 2019a. Jordania promete armonizar las leyes nacionales con compromisos internacionales y ampliar el apoyo a las mujeres y las niñas en muchos ámbitos (actualizado). http://www.unwomen.org/es/get-involved/step-itup/commitments/jordan [09/12/2019].

. 2019b. En Líbano, país de acogida, las mujeres refugiadas y campesinas 
fomentan la capacidad empresarial, la cohesión y el futuro. http://www.unwomen.org/es/news/stories/2016/9/in-lebanon-refugee-and-ruralwomen-build-entrepreneurship [09/12/2019].

2019c. Generan ganancias y cambian la mentalidad: las cooperativas de mujeres detrás del cambio social en los Territorios Palestinos Ocupados. http://www.unwomen.org/es/news/stories/2012/8/making-profits-changingmindsets-women-s-cooperatives-bring-social-change-in-the-occupied-palestin [09/12/2019].

2019d. Fondo para la Igualdad de Género. http://www.unwomen.org/es/trust-funds/fund-for-gender-equality [09/12/2019].

Pappé, I. 2014. The Modern Middle East. A Social and Cultural History. Third Edition. London and New York: Routledge.

Paradela Alonso, Nieves. 2015. "El feminismo árabe y su lucha por los derechos de la mujer". Feminismo/s, 26 (2015): 17-29.

Razzaz, Susan. 2017. A Challenging Market Becomes More Challenging: Jordanian Workers, Migrant Workers, and Refugees in the Jordanian Labour Market. International Labour Organization Office for Arab States. Beirut: ILO.

Stave, Svein Erik and Solveig Hillesund. 2015. Impact of Syrian Refugees on the Jordanian Labour Market: Findings from the Governorates of Amman, Irbid and Mafraq. Geneva: ILO y FAFO.

UNICEF. 2011a. ALGERIA: MENA Gender Equality Profile: Status of Girls and Women in the Middle East and North Africa. https://www.unicef.org/gender/ files/Algeria-Gender-Eqaulity-Profile-2011.pdf [09/12/2019].

. 2011b. JORDAN: MENA Gender Equality Profile: Status of Girls and Women in the Middle East and North Africa. http://www.unicef.org/gender/files/Jordan-Gender-Eqaulity-Profile-2011.pdf [09/ 12/2019].

2011c. MOROCCO: MENA Gender Equality Profile: Status of Girls and Women in the Middle East and North Africa. https://www.unicef.org/gender/files/Morroco-Gender-Eqaulity-Profile-2011.pdf [09/12/2019].

2011d. OCCUPIED PALESTINIAN TERRITORY MENA Gender Equality Profile: Status of Girls and Women in the Middle East and North Africa. http://www.unicef.org/gender/files/oPT-Gender-Eqaulity-Profile-2011.pdf [09/12/2019]. 
2011e. QATAR MENA Gender Equality Profile: Status of Girls and Women in the Middle East and North Africa. http://www.unicef.org/gender/files/Qatar-Gender-Eqaulity-Profile-2011.pdf [02/12/2019].

. 2011f. YEMEN: MENA Gender Equality Profile: Status of Girls and Women in the Middle East and North Africa. http://www.unicef.org/gender/files/Yemen-Gender-Eqaulity-Profile-2011.pdf [09/12/2019].

World Bank Group. 2019. The Little Data Book of Gender. Washington: International Bank for Reconstruction and Development. https://openknowledge.worldbank.org/bitstream/handle/10986/31689/LDB-ender2019.pdf? sequence $=2 \&$ isAllowed $=y[12 / 12 / 2019]$.

World Bank Group. 2018. Hashemite Kingdom of Jordan - Understanding How

Gender Norms in MNA Impact Female Employment Outcomes (English). Washington, D.C.: World Bank Group.

http://documents.worldbank.org/curated/en/859411541448063088/HashemiteKingdom-of-Jordan-Understanding-How-Gender-Norms-in-MNA-ImpactFemale-Employment-Outcomes

Páginas web:

https://www.cia.gov/library/publications/the-world-factbook/geos/le.html [12/12/ 2019].

Ciudad Mujer. Secretaría de Inclusión Social. 2017. Proyecto Fondo Mujeres. http://www.ciudadmujer.gob.sv/cooperacion-italiana-sis-onu-mujeres-y-bandesalpresentan-resultados-del-primer-ano-de-fondo-mujer/ [14/11/2019].

http://www.exteriores.gob.es/Documents/FichasPais/EMIRATOSARABESUNIDOS_F ICHA\%20PAIS.pdf [09/12/2019].

http://www.exteriores.gob.es/Documents/FichasPais/JORDANIA_FICHA\%20PAIS.pdf [09/12/2019].

http://www.exteriores.gob.es/Documents/FichasPais/MARRUECOS_FICHA\%20PAIS. pdf [09/12/2019].

http://www.exteriores.gob.es/Documents/FichasPais/OMAN_FICHA\%20PAIS.pdf [09/12/2019].

https://iraqiwomennet.wordpress.com [09/12/2019]. 
http://www.johud.org.jo/ [09/12/2019].

https://www.jordanriver.jo/en [09/12/2019].

http://jwu.org.jo/PagesDetiles.aspx?lng=1\&pageid=9 [09/12/2019].

http://www.musawah.org/ [09/12/2019].

http://www.nooralhusseinfoundation.org/ [09/12/2019].

http://www.women.jo [09/12/2019]. 